\title{
Prolonging Network Lifetime by Optimizing Actuators Deployment with Probabilistic Mutation Multi-layer Particle Swarm Optimization
}

\author{
Yamin Han ${ }^{1}$, Heejung Byun ${ }^{2 *}$, and Liangliang Zhang ${ }^{2}$ \\ ${ }^{1}$ Shandong Provincial Key Laboratory of Network \\ Based Intelligent Computing, University of Jinan, China \\ [e-mail: yaminhan11@gmail.com] \\ ${ }^{2}$ Department of Information and Telecommunications Engineering, \\ The University of Suwon, South Korea \\ [heejungbyun@suwon.ac.kr] \\ *Corresponding author: Heejung Byun
}

Received March 1, 2021; revised April 27, 2021; accepted June 8, 2021; published August 31, 2021

\begin{abstract}
In wireless sensor and actuator networks (WSANs), the network lifetime is an important criterion to measure the performance of the WSAN system. Generally, the network lifetime is mainly affected by the energy of sensors. However, the energy of sensors is limited, and the batteries of sensors cannot be replaced and charged. So, it is crucial to make energy consumption efficient. WSAN introduces multiple actuators that can be regarded as multiple collectors to gather data from their respective surrounding sensors. But how to deploy actuators to reduce the energy consumption of sensors and increase the manageability of the network is an important challenge. This research optimizes actuators deployment by a proposed probabilistic mutation multi-layer particle swarm optimization algorithm to maximize the coverage of actuators to sensors and reduce the energy consumption of sensors. Simulation results show that this method is effective for improving the coverage rate and reducing the energy consumption.
\end{abstract}

Keywords: Wireless sensor and actuator networks, actuators deployment, energy consumption, coverage, particle swarm optimization

A preliminary version of this paper was presented at ICONI 2020, and was selected as an outstanding paper.

This research was supported by Basic Science Research Program through the National Research Foundation of Korea (NRF) funded by the Ministry of Science, ICT \& Future Planning (NRF-2020R1A2C1004390). 


\section{Introduction}

$\mathbf{W}$ ireless sensor and actuator networks [1][2] consist of sensor nodes, actuator nodes, and sink nodes. Sensors with limited energy and short transmission range can collect and process data from the monitored region. Actuators with rich energy and longer transmission capabilities can collect and process data. Actuators can also interact with the monitoring environment. Additionally, sink node is used to aggregate data and monitor network systems. These nodes can connect and communicate with each other to monitor the environment and process events. WSANs have been widely applied into various fields such as intelligent agriculture, fire monitoring, and smart cities [3]-[6].

Network lifetime is an important criterion for evaluating network system services. The network lifetime is mainly affected by the energy of sensors. The energy consumed by sensors is mainly used for data transmission with sink nodes [7][8]. The energy consumption of sensors is directly related with the distance between the current sensor and the sink node. Additionally, the energy of sensors is limited, and the batteries of sensors cannot be replaced and charged. To increase the energy efficiency of sensors and extend the network lifetime, some researchers have proposed some technologies such as linear programming and sensor clustering routing protocol to extend the network lifetime [9][10]. However, when the monitored region becomes wide and the number of sensors is large, it becomes more difficult to prolong the network lifetime and increase the communication congestion. Therefore, it is crucial to save the energy of sensors to prolong the lifetime of the network in a larger monitored region.

WSAN introduces multiple actuators that can be regarded as multiple collectors to collect data from their respective surrounding sensors. However, the number of sensors is relatively large, and the number of actuators is limited in a WSAN system [11]. Therefore, it is necessary to research how to deploy a limited number of actuators according to the distribution of sensors to prolong the lifetime of the network.

To solve the problem of actuator deployment, thereby prolonging the network lifetime, this research optimizes actuator deployment using a probabilistic mutation multi-layer particle swarm optimization (PMMLPSO) algorithm. During the optimization process of actuator deployment, the coverage rate as well as the energy consumption of sensors are taken as optimization objectives. In this case, sensors can send data to the corresponding actuator and reduce the energy consumption. In addition, the PMMLPSO distributes the population into multiple layers and the particles mutate based on probability, which ensures the particles of lower layer learn better or different experiences from the subpopulations at the higher. This can increase the diversity of the searching and prevents falling into the local optima, thereby improving the performance of optimizing actuators deployment.

This work is structured as follows. Section 2 describes the problem and defines the WSAN system model. The optimization of actuator deployment with PMMLPSO is introduced in Section 3. The simulations are represented in Section 4. The conclusion of this paper is summarized in Section 5. 


\section{Related Works}

To increase the energy efficiency of sensors and extend the network lifetime, some studies have given relevant solutions. One of the studies regarded the problem of maximizing network lifetime as a linear programming problem [9][12][13]. The work in [12] pointed out that the energy consumption per unit of information transmission depends on the selection of the next-hop node, i.e., the selection of routing. Then they regarded the routing problem as a linear programming problem, which aims to maximize the network lifetime. Madan et al. adopted the subgradient algorithms to calculate the optimal routing scheme based on a distributed way, which can delay the time when the first sensor node runs out of its energy, thus prolonging the lifetime of the network [9]. To maximize network lifetime, the study in [13] considered the optimal scheduling of communication between the network and the base station as a linear programming problem, which makes the maximum life obtained by any combination of the chains shown as the upper limit.

Beyond that, some works adopted sensor clustering method to extend the network lifetime [10], [14-19]. Clustering of nodes is the most common technology for energy aware routing in WSN, and the most popular clustering protocol in WSN is low energy adaptive clustering hierarchy (LEACH) based on adaptive clustering technology, which is widely used in various fields [14]. To prolong the network lifetime and improve the network quality of service (QoS), the authors in [10] optimized the QoS parameters based on energy efficient inter cluster coordination protocol at the network layer level. The study in [15] improved the selection of cluster head based on clustering routing protocol, and avoided the sensors with low residual energy becoming cluster head, which can balance the energy consumption between sensors and prolong the network lifetime. The authors in [16] adopted fuzzy logic to realized cluster head selection and cluster formation. To reduce the chain-based energy consumption of cluster head selection and data routing based, the work in [17] proposed the multiple hop routing strategies (the shortest grid routing, partition super grid direct routing, and the shortest super grid routing) to prolong the network lifetime. The study in [18] proposed a distance and energy constrained k-means clustering scheme (DEKCS) to prolong the network lifetime. The cluster head was selected according to the position of the sensor in the cluster and the residual energy. The authors in [19] proposed a fuzzy-based two-level hierarchical routing protocol to enhance the lifetime of the network. For the protocol, the selection of cluster head and super cluster head was based on identifying the identification of appropriate parameters.

However, when the monitored region becomes wide and the number of sensors is large, it becomes more difficult to prolong the network lifetime and increase the communication congestion. WSAN introduces actuators, which can be regarded as multiple collectors gathering data from sensors in their respective regions instead of all cluster heads in WSN sending data to only one sink node. Nevertheless, how to deploy actuators according to the distribution of sensors need further research.

To find the optimal actuators deployment strategy based on the distribution of sensors and extend the network lifetime, the actuators deployment is optimized by the probabilistic mutation multi-layer particle swarm optimization (PMMLPSO) algorithm proposed in this paper. The coverage rate and energy consumption are regarded as optimization objectives in the process of actuators optimization. Furthermore, to avoid falling into the local optimum and increase the diversity of search ability, the proposed PMMLPSO method distributes the population in multiple layers, and the particles mutate based on probability, which ensures that 
the lower layer particles can learn better or different experiences from the subpopulations at the higher, thereby improving the performance of optimizing actuators deployment.

\section{Problem Description and System Modeling}

We assume $s$ sensors and $n$ actuators in the monitoring region $R$. All sensors are randomly scattered in $R$. Also, all sensors are homogeneous, i.e., all the sensors have same communication distance and sensor distance. Actuators can discover each other and collaborate with other actuators in the monitoring region through wireless communication, and they can also gather data from sensors within their respective coverage area.

\subsection{Energy Consumption Model}

Sensors consume a lot of energy to transmit data to actuators, the energy consumption of sending data adopts the energy dissipation model [20][21].

Sensors consume most of the energy $E$ in data transmission. $E$ is affected by both the distance $d_{s a}$ between the sensor and the corresponding actuator and the data length $L$. As shown in equation (1).

$$
E_{\mathrm{i}}=\left\{\begin{array}{l}
L^{*} E_{e}+L * E_{f} * d_{s a}^{2}, d_{s a}<d_{0} \\
L^{*} E_{e}+L * E_{m} * d_{s a}^{4}, d_{s a} \geq d_{0}
\end{array}\right.
$$

where $E_{i}$ is the energy consumption of the $i$-th sensor, $E_{e}$ is the dissipated energy in the electronic circuit of the radio model, $E_{f}$ and $E_{m}$ represent the free space and multipath dissipated energy in the amplifier of the radio model, respectively. $d_{0}$ is a constant and it can be calculated by equation (2).

$$
d_{0}=\sqrt{\frac{E_{f}}{E_{m}}}
$$

It can be observed from equation (1) that the energy consumption of the sensor to send data with $L$ length is only related to the distance $d_{s a}$, i.e., the distance between the current sensor and the corresponding actuator. When $d_{s a}$ is smaller than the distance threshold $d_{0}$, the consumed energy increases with the square of $d_{s a}$. In contrast, $d_{s a}$ is bigger than or equal to $d_{0}$, the consumed energy increases rapidly with $d_{s a}$. So, to reduce the energy consumption, the distance $d_{s a}$ must be minimized. $d_{s a}$ is given by equation (3).

$$
d_{s a}=\sqrt{\left(x_{s}-x_{a}\right)^{2}-\left(y_{s}-y_{a}\right)^{2}}
$$

where $\left(x_{s}, y_{s}\right)$ is the location of the sensor, $\left(x_{a}, y_{a}\right)$ is the location of the actuator.

All the consumed energy $E$ is shown in equation (4),

$$
E=\sum_{\mathrm{i}=1}^{s} E_{i}
$$


The energy consumption rate $E_{r}$ is shown in equation (5).

$$
E_{r}=\frac{E}{\sum_{i=1}^{s} E_{i}^{0}}
$$

where $E_{i}^{0}$ means the initial energy of the $i$-th sensor.

\subsection{Coverage Model}

For the coverage rate of actuators to sensors, the detection probability model [22] is adopted to estimate whether a sensor is covered by actuators. Coverage problem refers to covering sensors with the communication region of actuators. Specifically, the actuator and the sensor have their corresponding communication range, if sensors and one actuator can communicate effectively, this represents that the sensors are within the coverage of the communication area of the actuator. The communication radius of actuators is $r_{a}$. The coverage probability model is used to estimate whether a sensor $s_{j}$ is covered by an actuator $a_{i}$. The probability $P\left(a_{i}, s_{j}\right)$ that $s_{j}$ is covered by $a_{i}$ is based on the distance between $a_{i}$ and $s_{j}$, which can be expressed by equation (6).

$$
P_{\left(a_{i}, s_{j}\right)}=\left\{\begin{array}{c}
1, r_{a}-r_{e} \geq d_{a_{i} s_{j}} \\
\exp \left(-\alpha_{1} \frac{\lambda_{1}^{\beta_{1}}}{\lambda_{2}^{\beta_{2}}}+\alpha_{2}\right), r_{a}-r_{e}<d_{a_{i} s_{j}}<r_{a}+r_{e} \\
0, r_{a}+r_{e} \leq d_{a_{i} s_{j}}
\end{array}\right.
$$

where $d_{a_{i}, s_{j}}$ is the distance between the $i$-th actuator $a_{i}$ and the $j$-th sensor $s_{j}, r_{e}$ is the coverage error range and $r_{e}<r_{a} . \alpha_{1}, \alpha_{2}, \beta_{1}$, and $\beta_{2}$ are parameters of coverage probability. In addition, $\lambda_{1}=r_{e}-r_{a}+d_{a_{i}, s_{j}}$ and $\lambda_{2}=r_{e}+r_{a}-d_{a_{i}, s_{j}}$.

The coverage probability $P_{s_{j}}^{\prime}$ that a sensor $s_{j}$ can be covered is calculated by equation (7).

$$
P_{\left(s_{j}\right)}^{\prime}=1-\prod_{i=1}^{n}\left(1-P_{\left(a_{i}, s_{j}\right)}\right)
$$

The total of coverage rate is calculated by equation (8),

$$
C=\sum_{j=1}^{s} \frac{P_{\left(s_{j}\right)}^{\prime}}{m}
$$

Actuators collect data from sensors within their respective communication ranges, and then send the collected data to sink. The communication between sensors and the corresponding actuator adopts TDMA schedule [23]. First, each actuator makes a TDMA schedule and sends its TDMA schedule to their sensor members. Then, each sensor sends data to the actuator according to the TDMA schedule. Finally, actuators send the data gathered from their sensor members to sink node. In addition, if one sensor is covered by more actuators, the sensor sends data to the actuator closest to it. 


\section{Optimizing Actuator Deployment with Probabilistic Mutation Multi-layer Particle Swarm Optimization}

\subsection{Standard Particles Swarm Optimization}

The optimization method of PSO originated from the behavior of birds flocking, it can build a dynamic model for the problem to be optimized. The inertia weight $w$ was introduced into the original PSO algorithm, which can be regarded as standard PSO (SPSO) [24]. Each particle includes two variables: position and velocity. One particle means a potential solution for an optimization problem. All particles flying in a $D$-dimensional search space during optimization, the flying distance and direction are based on the best solution Pbest found by itself and the optimal solution found by the population Gbest. The update of position $x_{i}$ and velocity $v_{i}$ can be calculated as equation (9) and equation (10), respectively.

$$
\begin{gathered}
v_{i}=w v_{i}+r c_{1}\left(\text { Gbest }-x_{i}\right)+r c_{2}\left(\text { Pbest }-x_{i}\right) \\
x_{i}=x_{i}+v_{i}
\end{gathered}
$$

where $r$ is randomly generated within $[0,1] . c_{1}$ and $c_{2}$ are the acceleration coefficients.

PSO is widely applied to find the optimal or near-optimal solution to the problem. However, for the high dimensional and more complex problems, traditional PSO is easy to converge to local optimum.

\subsection{PMMLPSO: Probabilistic Mutation Multi-layer Particle Swarm Optimization}

Probabilistic Mutation Multi-layer Particle Swarm Optimization

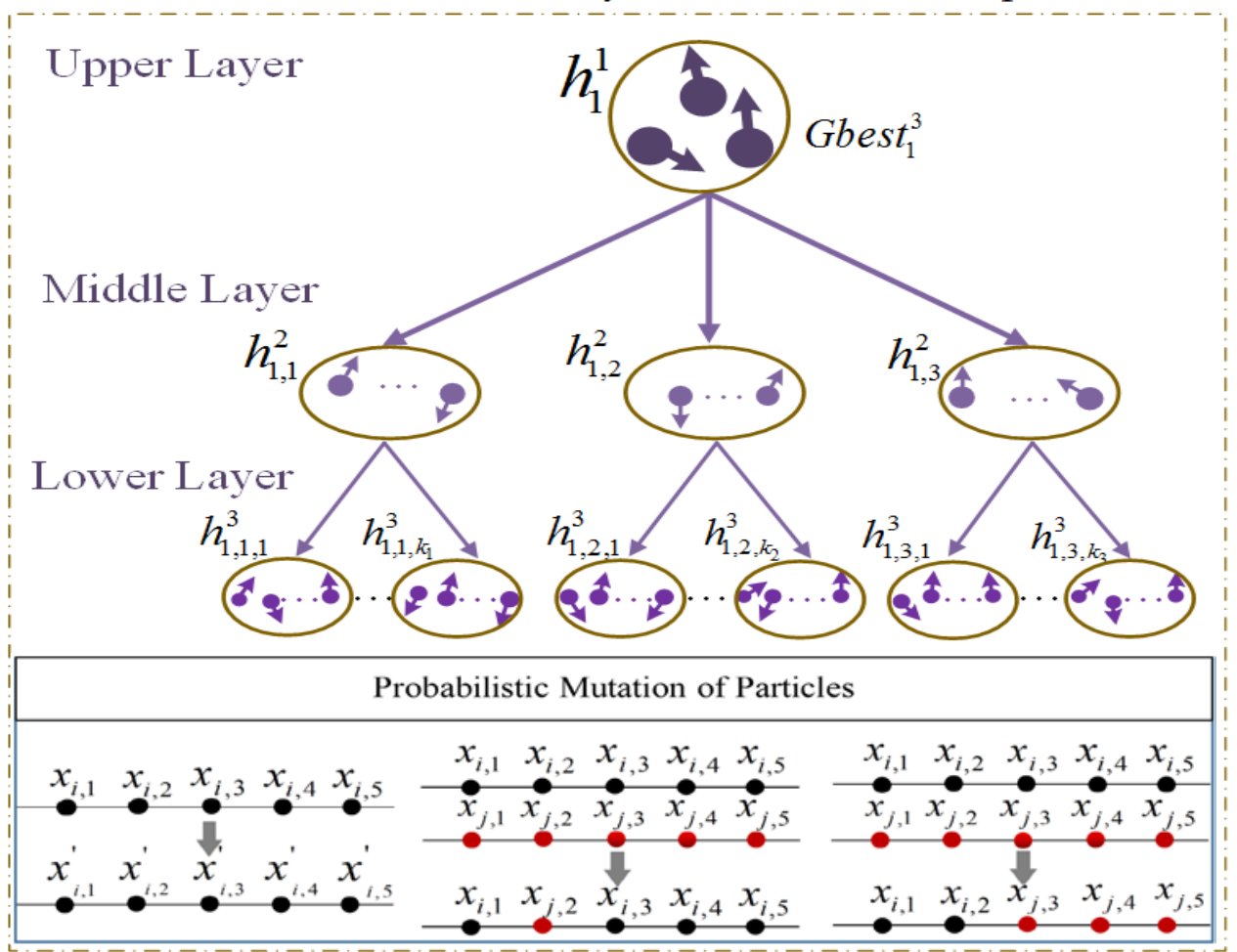

Fig. 1. An example of a three-layer structure and particle mutation of the proposed PMMLPSO. 
To effectively optimize the deployment of actuators, a PMMLPSO based on a multi-layer structure [25] and probabilistic mutation is proposed to increase the diversity of the searching. The proposed PMMLPSO method is depicted in Fig. 1. The two layers architecture of traditional PSO is increased to the multi-layer architecture. The particles in the higher layer are composed of particles in the lower layer, and particles in the layers higher than the first layer can also be called subpopulations. The particles of lower layer learn better or different experiences from the subpopulations at the higher. In addition, particles mutate based on probability. For instance, increasing or decreasing the value of different dimensions of particles based on probability, transforming the value of one dimension of the current particle based on another particle, and transforming the value of several continuous dimensions of the current particle based on another particle.

The multi-layer strategy and particle mutation scheme can help increase the diversity of searching for optimal solutions and prevent falling into local optima, so as to improve the performance of PSO to optimize actuator deployment. For the proposed PMMLPSO method, the velocity of the particle is updated according to the optimal particle of all layers containing the current particle, and the velocity update formula of the $i$-th dimension of the particle as shown in equation (11).

$$
v_{i}=w v_{i}+\sum_{j=1}^{m} c_{j} r\left(\text { Pbest }_{i, j}-x_{i}\right), c_{j}=\frac{c}{m}
$$

where $m$ means the number of layers of the particle swarm, $c$ is the acceleration constant.

\subsection{Optimizing the Actuators Deployment with PMMLPSO}

We assume that there are $n$ actuators in the WSAN system, then $2 n$ parameters are optimized, and two adjacent parameters correspond to the location of one actuator. A particle can be encoded by $\left(x_{1}, y_{1}, x_{2}, y_{2}, \ldots, x_{n}, y_{n}\right)$, where $\left(x_{j}, y_{j}\right)$ is the position of the $j$-th actuator, as shown in Fig. 2.

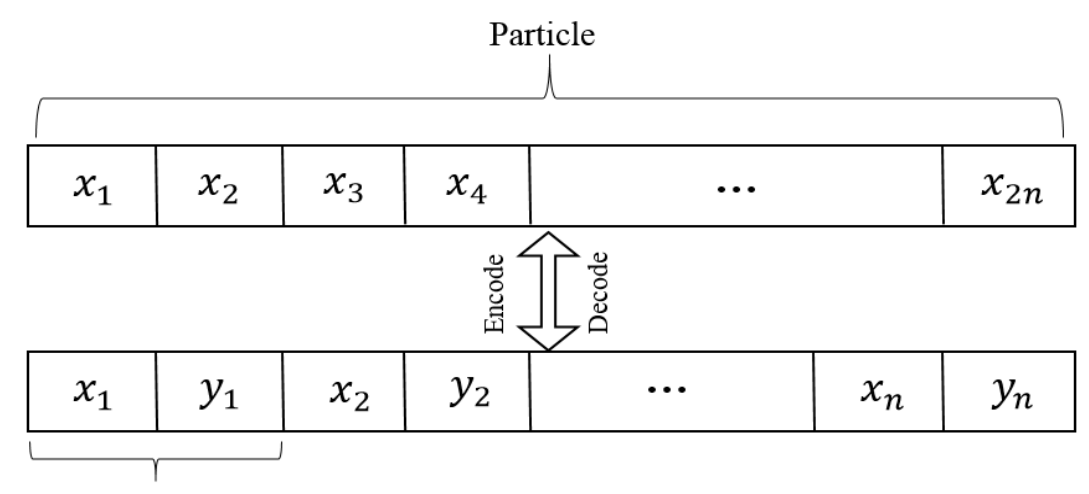

Position of the first actuator

Fig. 2. Composition of particles

In addition, the goal of this work is to optimize the actuators deployment, taking into account both the energy consumption and the coverage rate. The fitness function of the optimization process for the actuator deployment can be expressed as shown in equation (12). 


$$
F=\varepsilon_{1} C+\varepsilon_{2}\left(1-E_{r}\right)
$$

where $\varepsilon_{1}$ and $\varepsilon_{2}$ are the weights in the optimization process of coverage rate and energy

consumption, and $\varepsilon_{1}+\varepsilon_{2}=1$.

From equation (12) we can observe that the fitness value of each particle is expected to be as large as possible. The optimization process of the proposed PMMLPSO method for actuator deployment can be summarized as follows:

(1) Define the number of particles, layers, iterations, and some other parameters of the PMMLPSO method.

(2) Initialize the dimension of each particle as well as initialize the velocity and position of each particle. The dimension of particles is twice the number of actuators, and each particle corresponds to a different deployment position of the actuators, i.e., each particle represents a different actuator deployment solution.

(3) All particles update their respective velocity and position in each iteration until the termination condition is met. In each iteration, the particles of lower layer learn better or different experiences from the subpopulations at the higher. Also, each particle mutates based on probability. This can help increase the diversity of the searching and prevents falling into the local optima.

(4) Finally, the solution represented by the particle with the highest fitness value is regarded as the final actuator deployment solution.

According to this solution, the actuators are deployed among the sensor, as shown in Fig. 3. It can be observed that the deployment of actuators takes into account the distribution of sensors, i.e., actuators are only deployed in subregions where sensors are distributed, and there are no actuators in non-sensors subregions, which effectively saves resources.

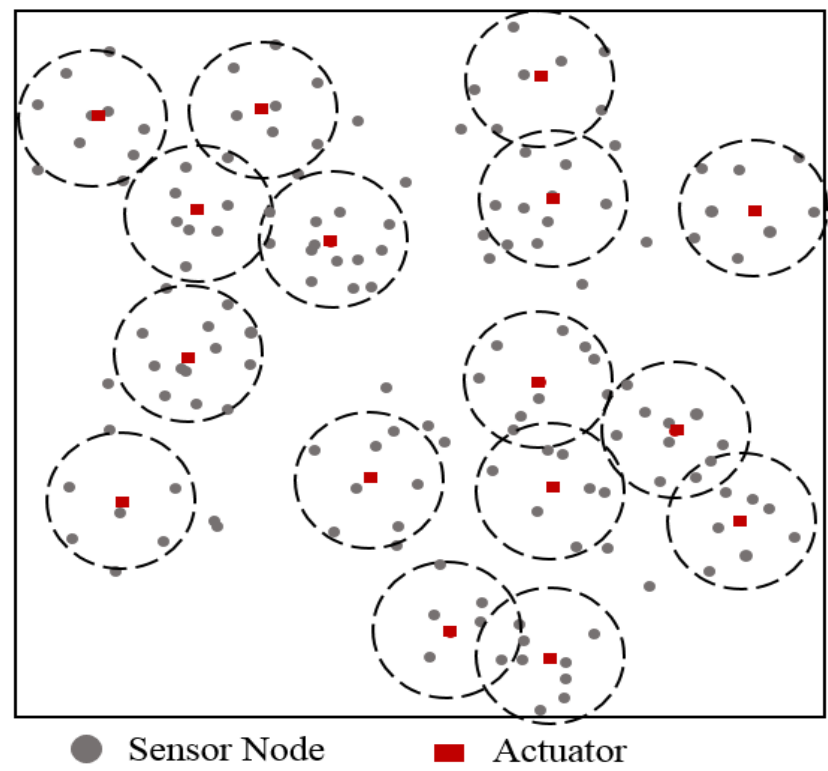

Fig. 3. Actuators deployment based on sensors distribution 
The above process of using PMMLPSO to optimize actuators deployment can be described as Algorithm 1.

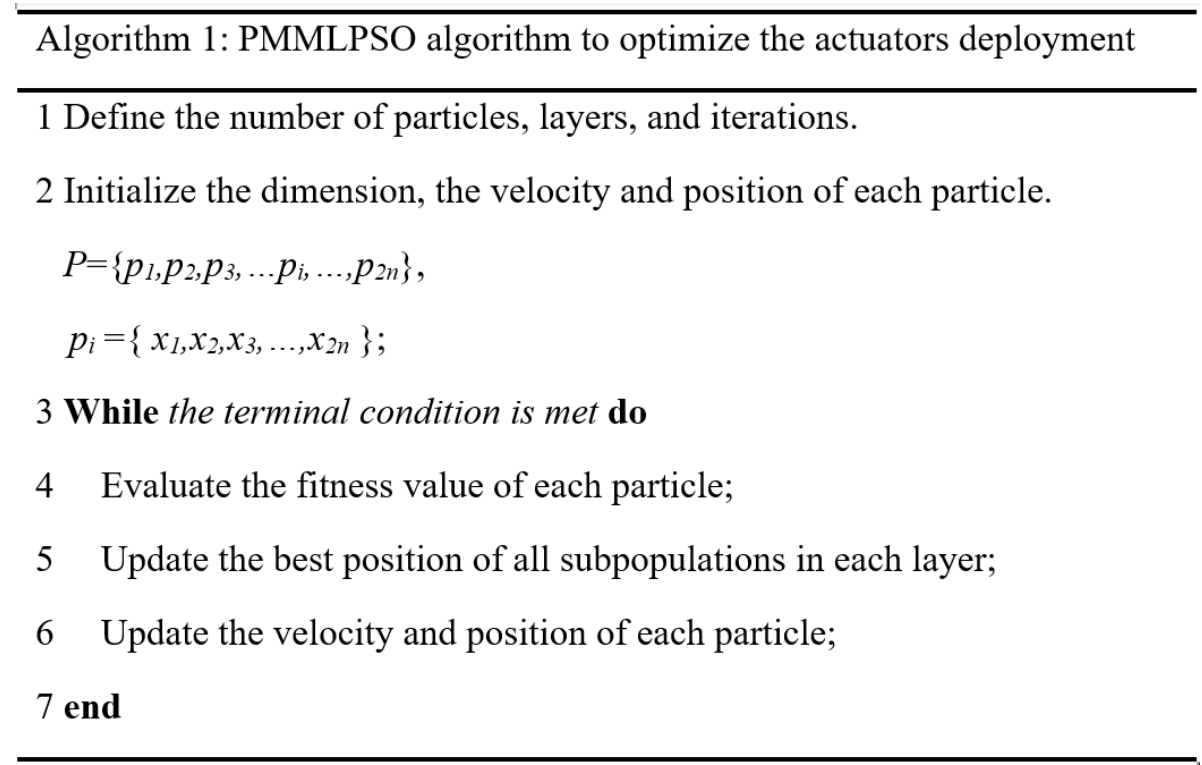

\section{Simulations and Results}

To corroborate the performance of PMMLPSO method for the deployment of actuators in WSAN, the simulation is done using MATLAB. PMMLPSO method is compared with the standard PSO (SPSO) [24] and some variants of SPSO such as dynamic multi-swarm particle swarm optimizer (DMS-PSO) [26], multi-layer particle swarm optimization (MLPSO) [25], nonlinear fitness-based inertia weight PSO (NFIWPSO) [27], and the PSO method with adjusted inertial weight (AIWPSO) [28].

For SPSO, the inertia weight was introduced into the original PSO algorithm, this can adjust the impact of the previous generation particle velocity on the current particle velocity. DMS-PSO improved the performance of the original PSO by dynamically combining particles into several groups in the iterative process. MLPSO adjusted the two-layer structure of the original PSO into multiple layers to add the diversity of the population. NFIWPSO dynamically updated inertia weights based on nonlinear fitness values and AIWPSO adjusted the inertia weight according to the number of iterations.

The parameter settings in the simulations are as below: 300 sensors are randomly scattered in a two-dimensional monitoring region of 400 meters $\times 400$ meters. For all methods, the number of iterations is 1000 , the number of particles is 64 , the value of parameters of the compared related methods are set to their recommended values, and the number of actuators is set to 20 . The average of 30 simulation results is taken as the final result of each method to reduce the statistical error.

In this section, four sets of simulations have been carried out to assess the significance of the proposed PMMLPSO method, and to compare the PMMLPSO method with related algorithms. In the first set of simulations, the coverage rate is compared in the process of actuator deployment optimization. Then, the energy cost rate is compared. After that, the fitness value 
is compared during actuators deployment optimization. Finally, the comparison of optimization results on coverage rate and energy consumption rate are given.

\subsection{Comparison of Coverage Rate During Actuator Deployment Optimization}

Coverage refers to the coverage of actuators to sensors, and the coverage rate refers to the ratio of the number of sensors covered by the actuator to the number of all sensors. In the WSAN, the number of sensors is large while the number of actuators is limited. It is meaningful to optimize the actuators deployment according to the distribution of sensors. This allows the sensor to send data to the actuator closer to it, and the actuator aggregates the data collected from the sensors within its coverage. Finally, the actuator transmits the data to sink node. This can effectively avoid the energy hole problem and extend the network lifetime. Thus, the coverage rate simulation of PMMLPSO method and other related methods is conducted.

Fig. 4 shows the coverage rate convergence curve during the optimization of actuator deployment. It can be noticed that SPSO, AIWPSO, and NFIWPSO get lower coverage rate. Because these methods tend to converge to the local optimum. In the process of searching for the optimal or near optimal solution of actuator deployment, the DMSPSO method is difficult to converge due to the randomness of searching. MLPSO method can converge to the better value of coverage rate earlier. However, the proposed PMMLPSO method in this work obtains the highest coverage rate. Since the PMMLPSO method adopts a multi-layer architecture and a probability-based mutation strategy, the particles can learn experience from different layers and increase the diversity of the population. This effectively improves the search ability of particles for the optimal or near optimal solution. Therefore, the above simulation proves the effectiveness of the proposed PMMLPSO method to optimize actuator deployment.

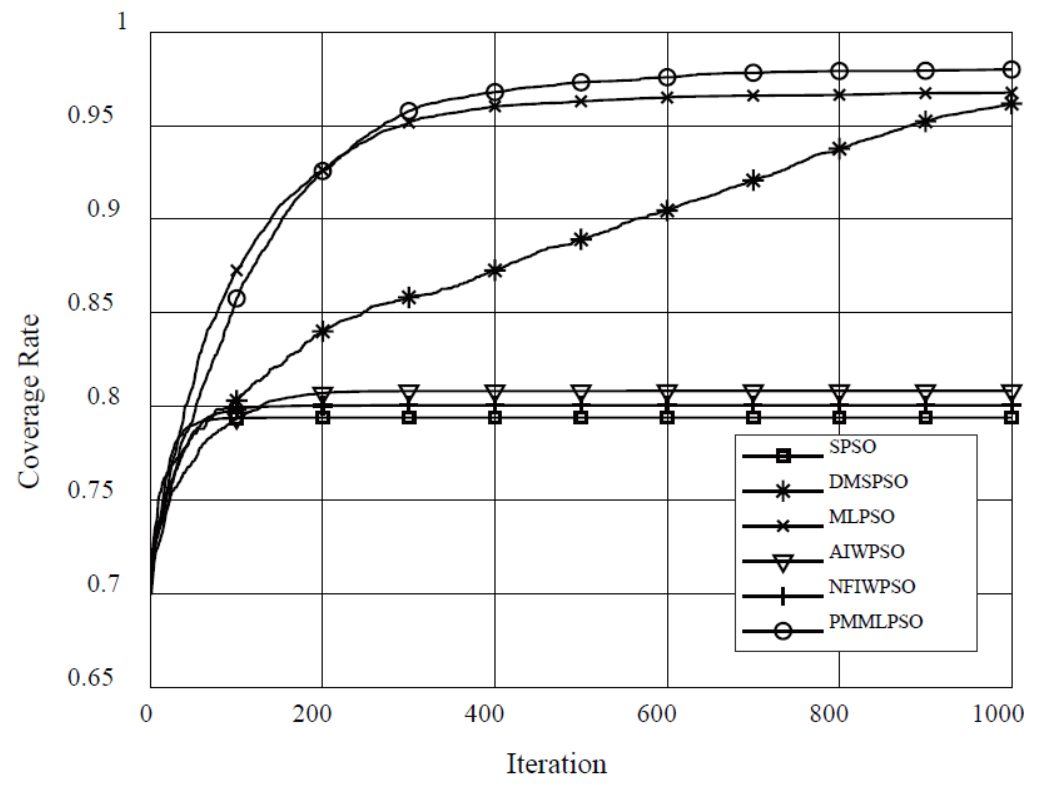

Fig. 4. Comparison of coverage rate during actuators deployment optimization.

\subsection{Comparison of Energy Cost Rate During Actuator Deployment Optimization}

The lifetime of network is affected by the energy of sensors. Most of energy consumed by sensors is in data transmission. Furthermore, the energy consumption of sensors to transmit 
certain data is proportional to the transmission distance. Multiple actuators are deployed in the sensors, and the sensors can send data to the closer actuator instead of sending it to sink node by one hop or multiple hops. This effectively reduces the energy consumption of the sensor. The energy consumption rate can well reflect whether actuators are deployed properly. So, the simulation of the energy consumption rate is conducted.

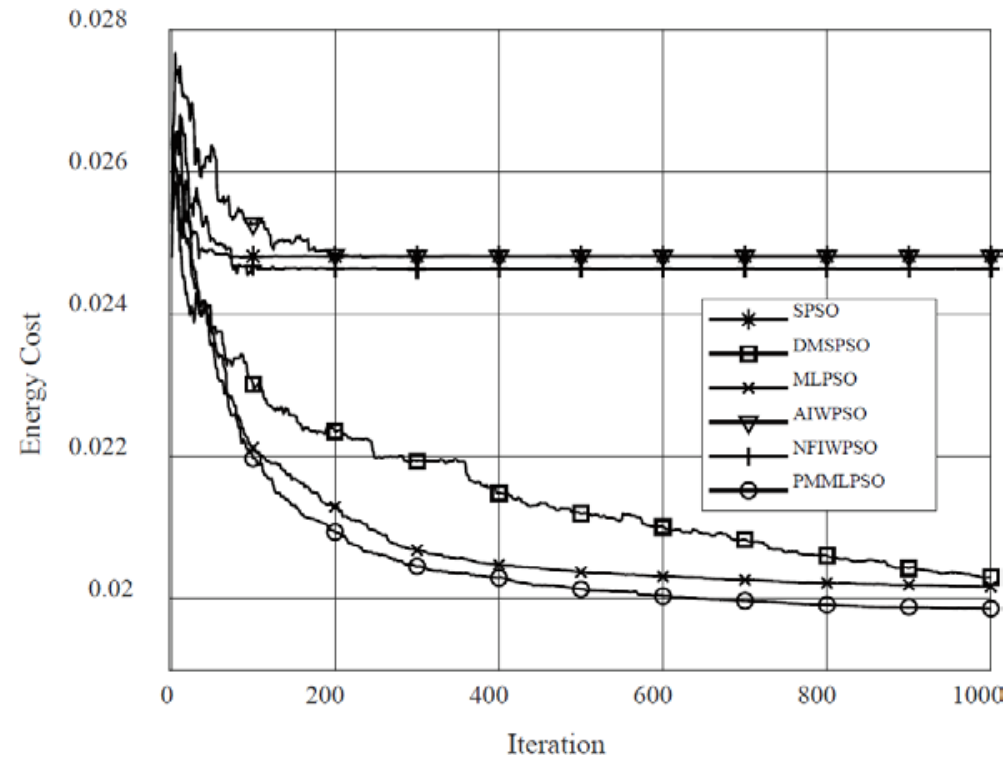

Fig. 5. Comparison of energy cost rate during actuators deployment optimization.

Fig. 5 shows the comparison of energy cost rate during the actuators deployment optimization. It can be observed that the SPSO, AIWPSO, and NFIWPSO have the highest energy consumption rate. Because these methods prematurely converge into the local optimum. On the contrary, MLPSO, DMSPSO, and PMMLPSO correspond to the lower energy consumption. Due to the randomness of DMSPSO, it is difficult to converge and achieve a lower energy consumption rate after reaching the same iteration times as MLPSO and PMMLPSO methods. Furthermore, the PMMLPSO proposed in this work has the lowest energy consumption rate, because the multi-layer structure and the probability-based variation scheme increase the optimization performance of PMMLPSO. Therefore, the proposed PMMLPSO shows superior performance in optimizing actuator deployment.

\subsection{Comparison of Fitness Value During Actuator Deployment Optimization}

The fitness value guides the optimization direction of the optimization algorithms for the actuator deployment, and it also reflects the quality of the current solutions. In this section, the simulation of the convergence curve of fitness in the process of actuator deployment optimization is organized.

Fig. 6 shows the convergence results obtained by PMMLPSO and other methods for optimizing the deployment of actuators. The proposed PMMLPSO method has the highest fitness value, this indicates that the PMMLPSO method obtains the highest coverage rate and the lowest energy consumption rate for the actuator optimization of WSAN. Since particles of PMMLPSO learning experience from multi-layer, which can help increase the diversity of searching for optimal solutions. In addition, the probabilistic mutation scheme can help 
prevent falling into local optima. However, particles of some methods only learn experience of two layers, i.e., the best experience of the current particle and the best experience of the entire swarm. For instance, SPSO, NFIWPSO, and AIWPSO, they are easy to converge to local optimum. In addition, strong randomness difficult to help DMSPSO search for good solutions, this slows its convergence speed. Overall, the proposed PMMLPSO outperforms its competitors and shows higher performance in optimizing the deployment of actuators.

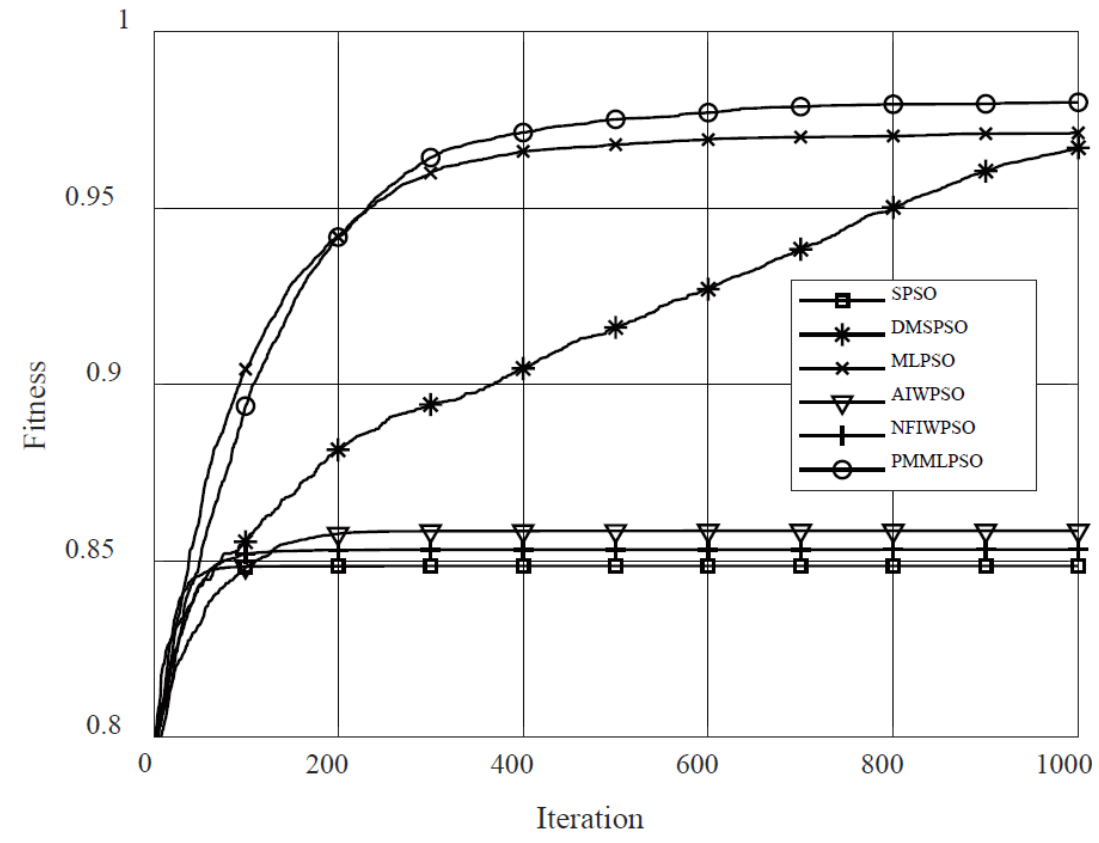

Fig. 6. Comparison of fitness during actuator deployment optimization.

\subsection{Comparison of Optimization Results on Coverage Rate and Energy Consumption Rate}

After optimizing the deployment of actuators, a final optimal solution or near optimal solution is acquired. Actuators are deployed based on this solution, and the coverage rate and energy consumption rates obtained by PMMLPSO method and other related methods are compared in this section.

Table 1 describes the coverage rate and energy consumption rate obtained by SPSO, DMSPSO, MLPSO, AIWPSO, NFIWPSO, and PMMLPSO when optimizing actuator deployment. It can be seen that the original SPSO gets the lowest coverage rate and the highest energy consumption rate. This is because it easily converges to the local optima. All variants of SPSO have improved the performance of the original SPSO. However, AIWPSO and NFIWPSO are similar to SPSO in that they both have low coverage and high energy consumption, because particles learn best experience found by itself and the optimal experience found by the population based on two-layer architecture, which leads to convergence to local optimal. Among all the methods, the PMMLPSO proposed in this work gets the highest coverage rate and the lowest energy consumption rate. Since particles can learn experience from multiple layers and particles can mutate based on probability, this increases the diversity of searching and prevents particles from falling into local optima. Therefore, the proposed PMMLPSO method can effectively optimize the actuator 
deployment.

However, when the problem to be optimized is high-dimensional and complex, that is, when the deployment of a large number of actuators is optimized, the proposed PMMLPSO method faces similar limitations with other PSO based methods, i.e., it is easy to converge into the local optimum.

Table 1. The final comparison results on coverage rate and energy consumption rate

\begin{tabular}{|c|c|c|}
\hline Methods & Coverage Rate & Energy Consumption Rate \\
\hline SPSO & $79.42 \%$ & $2.48 \%$ \\
\hline DMSPSO & $96.10 \%$ & $2.03 \%$ \\
\hline MLPSO & $96.76 \%$ & $2.02 \%$ \\
\hline AIWPSO & $80.84 \%$ & $2.48 \%$ \\
\hline NFIWPSO & $80.08 \%$ & $2.46 \%$ \\
\hline PMMLPSO & $\mathbf{9 7 . 9 9 \%}$ & $\mathbf{1 . 9 8} \%$ \\
\hline
\end{tabular}

\section{Conclusions}

In this work, the optimization strategy of actuator deployment is studied to expand the coverage of the actuators to sensors and reduce the energy consumption rate of sensors. To effectively optimize actuator deployment, a novel PMMLPSO is proposed. During the optimization process of actuators deployment, both the coverage rate and the energy consumption rate are considered. Simulations and results reveal that the PMMLPSO performs well in optimizing actuators deployment for WSAN, which effectively increases the coverage rate and decreases the energy consumption.

\section{References}

[1] N. Primeau, R. Falcon, R. Abielmona, and E. M. Petriu, "A review of computational intelligence techniques in wireless sensor and actuator networks," IEEE Communications Surveys Tutorials, 20(4), 2822-2854, 2018. Article (CrossRef Link).

[2] A. Nayak and I. Stojmenovic., Wireless sensor and actuator networks: algorithms and protocols for scalable coordination and data communication, John Wiley \& Sons, 2010. Article (CrossRef Link).

[3] N. Sabri, S. A. Aljunid, R. Ahmad, M. Malek, A. Yahya, R. Kamaruddin, and M. Salim, "Smart prolong fuzzy wireless sensor-actor network for agricultural application," Journal of Information Science and Engineering, vol. 28, no. 2, pp. 295-316, 2012. Article (CrossRef Link).

[4] C. Lu, A. Saifullah, B. Li, M. Sha, H. Gonzalez, D. Gunatilaka, C. Wu, L. Nie, and Y. Chen., "Real-time wireless sensor-actuator networks for industrial cyber-physical systems," Proceedings of the IEEE, vol. 104, no. 5, pp. 1013-1024, 2016. Article (CrossRef Link).

[5] X. Bai, L. Liu, M. Cao, J. Panneerselvam, Q. Sun, and H. Wang, "Collaborative actuation of wireless sensor and actuator networks for the agriculture industry,” IEEE Access, 5, 13286-13296, 2017. Article (CrossRef Link).

[6] H. Afzaal and N. A. Zafar, "Robot-based forest fire detection and extinguishing model," in Proc. of 2016 2nd International Conference on Robotics and Artificial Intelligence (ICRAI), pp. 112-117, Nov 2016. Article (CrossRef Link). 
[7] Giuseppe Anastasi, Marco Conti, Mario Di Francesco, and Andrea Passarella, "Energy conservation in wireless sensor networks: A survey,” Ad hoc networks, 7(3), 537-568, 2009. Article (CrossRef Link).

[8] Mariam Alnuaimi, Khaled Shuaib, Klaithem Alnuaimi, and Mohammed Abdel-Hafez, "Data gathering in delay tolerant wireless sensor networks using a ferry,” Sensors, 15(10), 25809-25830, 2015. Article (CrossRef Link).

[9] R. Madan and S. Lall, "Distributed algorithms for maximum lifetime routing in wireless sensor networks,” in Proc. of IEEE Global Telecommunications Conference, 2004. Article (CrossRef Link).

[10] Shalli Rani, Jyoteesh Malhotra, and Rajneesh Talwar, "On the development of realistic cross layer communication protocol for wireless sensor networks,” Wireless Sensor Network, 6(05), 57-66, 2014. Article (CrossRef Link).

[11] M. Zhao, Y. Yang and C. Wang, "Mobile Data Gathering with Load Balanced Clustering and Dual Data Uploading in Wireless Sensor Networks,” IEEE Transactions on Mobile Computing, vol. 14, no. 4, pp. 770-785, 1 April 2015. Article (CrossRef Link).

[12] Jae-Hwan Chang and L. Tassiulas, "Maximum lifetime routing in wireless sensor networks," IEEE/ACM Transactions on Networking, 12(4), 609-619, Aug 2004. Article (CrossRef Link).

[13] V. Tabus, D. Moltchanov, Y. Koucheryavy, I. Tabus and J. Astola, "Energy efficient wireless sensor networks using linear-programming optimization of the communication schedule,” Journal of Communications and Networks, vol. 17, no. 2, pp. 184-197, April 2015. Article (CrossRef Link).

[14] Sudhanshu Tyagi and Neeraj Kumar, "A systematic review on clustering and routing techniques based upon LEACH protocol for wireless sensor networks," Journal of Network and Computer Applications, 36(2), 623-645, 2013. Article (CrossRef Link).

[15] Xu Xia, Zhigang Chen, Deng Li, and Wanghuai Li, "Proposal for efficient routing protocol for wireless sensor network in coal mine goaf,” Wireless personal communications, 77(3), 1699-1711, 2014. Article (CrossRef Link).

[16] S. Lata, S. Mehfuz, S. Urooj and F. Alrowais, "Fuzzy Clustering Algorithm for Enhancing Reliability and Network Lifetime of Wireless Sensor Networks,” IEEE Access, vol. 8, pp. 66013-66024, 2020. Article (CrossRef Link).

[17] U. M. Durairaj and S. Selvaraj, "Two-Level Clustering and Routing Algorithms to Prolong the Lifetime of Wind Farm-Based WSN,” IEEE Sensors Journal, vol. 21, no. 1, pp. 857-867, 1 Jan.1, 2021. Article (CrossRef Link).

[18] K. G. Omeke et al., "DEKCS: A Dynamic Clustering Protocol to Prolong Underwater Sensor Networks,” IEEE Sensors Journal, vol. 21, no. 7, pp. 9457-9464, 1 April1, 2021. Article (CrossRef Link).

[19] D. U. Maheswari, S. Sudha and M. Meenalochani, "Fuzzy based adaptive clustering to improve the lifetime of wireless sensor network,” China Communications, vol. 16, no. 12, pp. 56-71, Dec. 2019. Article (CrossRef Link).

[20] Jorio, Ali, et al., "An energy-efficient clustering routing algorithm based on geographic position and residual energy for wireless sensor network," Journal of Computer Networks and Communications, vol. 2015, 2015. Article (CrossRef Link).

[21] Y. Han, B. Yang and H. Byun, “Optimizing Actuators Deployment for WSAN Using Hierarchical Intermittent Communication Particle Swarm Optimization,” IEEE Sensors Journal, vol. 21, no. 1, pp. 847-856, 1 Jan.1, 2021. Article (CrossRef Link).

[22] E. Onur, C. Ersoy, H. Delic, and L. Akarun, "Surveillance wireless sensor networks: Deployment quality analysis,” IEEE Network, 21(6), 48-53, November 2007. Article (CrossRef Link).

[23] M. Abo-Zahhad, S. M. Ahmed, N. Sabor and S. Sasaki, "Mobile Sink-Based Adaptive Immune Energy-Efficient Clustering Protocol for Improving the Lifetime and Stability Period of Wireless Sensor Networks,” IEEE Sensors Journal, vol. 15, no. 8, pp. 4576-4586, Aug. 2015. Article (CrossRef Link). 
[24] Y. Shi and R. Eberhart, “A modified particle swarm optimizer," in Proc. of IEEE International Conference on Evolutionary Computation Proceedings. IEEE World Congress on Computational Intelligence (Cat. No.98TH8360), pp. 69-73, May 1998. Article (CrossRef Link).

[25] Wang Lin, Yang Bo, and Yuehui Chen, "Improving particle swarm optimization using multi-layer searching strategy,” Information Sciences, 274(8), 70-94, 2014. Article (CrossRef Link).

[26] J. J. Liang and P. N. Suganthan, "Dynamic multi-swarm particle swarm optimizer," in Proc. of 2005 IEEE Swarm Intelligence Symposium, pp. 124-129, June 2005. Article (CrossRef Link).

[27] A. El Assaf, S. Zaidi, S. Affes, and N. Kandil, "Optimal anchors placement strategy for super accurate nodes localization in anisotropic wireless sensor networks," in Proc. of 2016 International Wireless Communications and Mobile Computing Conference (IWCMC), pp. 25-30, 2016. Article (CrossRef Link).

[28] Y. Zhou, N. Wang, and W. Xiang, "Clustering hierarchy protocol in wireless sensor networks using an improved PSO algorithm,” IEEE Access, 5, 2241-2253, 2016. Article (CrossRef Link).

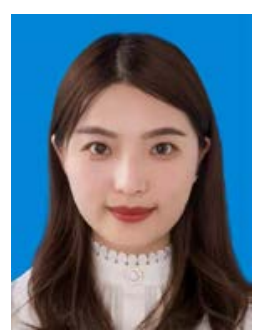

Yamin Han: She received the M.S. degree from the University of Jinan, China, in 2017, and the Ph.D. degree from the University of Suwon, South Korea, in 2021. Her current research interests include network protocol design, network modeling, intelligent computing, and machine learning.

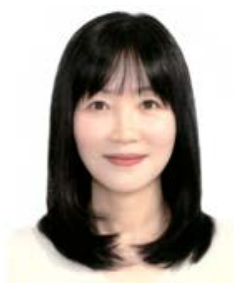

Heejung Byun: She received the B.S. degree from Soongsil University, South Korea, in 1999, and the M.S. and Ph.D. degrees from the Korea Advanced Institute of Science and Technology (KAIST), South Korea, in 2001 and 2005, respectively. She was a Senior Researcher with the Samsung Advanced Institute of Technology and Samsung Electronics Company Ltd. from 2007 to 2010. She is currently an Associate Professor with the Department of Information and Telecommunications Engineering, The University of Suwon, South Korea. Her research interests include network protocol design, network modeling, controller design, and theoretical analysis.

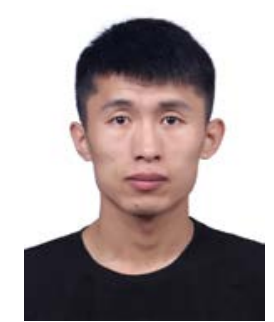

Liangliang Zhang: He received the B.S. and M.S. degrees from the University of Jinan, China, in 2015 and 2018, respectively. He is now pursuing the Ph.D. degree from the Department of Computer Science, The University of Suwon, Hwaseong, South Korea. His research interests include computational intelligence, fuzzy neural networks, pattern recognition, and image processing. 